\title{
Ruin Probabilities in the Mixed Claim Frequency Risk Models
}

\author{
Zhao Xiaoqin $^{1}$ and Chuangxia Huang ${ }^{1,2}$ \\ ${ }^{1}$ School of Mathematics and Computing Science, Changsha University of Science and Technology, Changsha 410004, China \\ ${ }^{2}$ Academy of Mathematics and Systems Science, Chinese Academy of Science, Beijing 10090, China \\ Correspondence should be addressed to Chuangxia Huang; cxiahuang@126.com
}

Received 26 December 2013; Revised 30 April 2014; Accepted 30 April 2014; Published 29 May 2014

Academic Editor: Fenghua Wen

Copyright (C) 2014 Z. Xiaoqin and C. Huang. This is an open access article distributed under the Creative Commons Attribution License, which permits unrestricted use, distribution, and reproduction in any medium, provided the original work is properly cited.

We consider two mixed claim frequency risk models. Some important probabilistic properties are obtained by probability-theory methods. Some important results about ruin probabilities are obtained by martingale approach.

\section{Introduction}

Mixture models are a fundamental tool in applied statistics, for most mixture models, including the widely used mixtures of Gaussians and hidden Markov models (HMMs); the current practice relies on the Expectation-Maximization (EM) algorithm, a local search heuristic for maximum likelihood estimation; an efficient method of moments approach to parameter estimation for a broad class of high-dimensional mixture models with many components was developed [13]. Finite mixture models have a long history in statistics; a detailed review of mixture models and models-based clustering was provided in [4], for a finite mixture of regressions model, $[5,6]$ develop an efficient EM algorithm for numerical optimization with provable convergence properties.

In this paper we consider two mixture models. In Section 2 we set up the binomial-Poisson model, and some important results about ruin probabilities are obtained by martingale approach.

In Section 3 we also set up another Poisson-dualistic model; in this section we obtain some important probabilistic properties and estimates for ruin probability.

In the classical risk model, the surplus of an insurance company over the interval $(0, t]$ is $X(t)$, which is defined by

$$
X(t)=c t-\sum_{k=1}^{N(t)} Z_{k}
$$

where $N(t)$ is to be interpreted as the number of claims on the company during the interval $(0, t]$; assume that $\{N(t) ; t \geq 0\}$ is an homogeneous Poisson process with intensity $\lambda$. In the complex problems of actual operation in insurance business, insurance company classifies the risk by its some characteristics, but the claim frequency $\lambda$ of the individual policy which has been classified into the same kind of portfolio may be different; that is, this is the so-called nonhomogeneity. For a nonhomogeneous portfolio, we can assume that $\lambda$ is a random variable; thus the mixed Poisson distribution model can be derived.

In general, just as reported in [6] if the number for claim $N$ is a discrete distribution with parameter $\alpha$ and the distribution sequence is

$$
P(N=k \mid \alpha)=p(k ; \alpha)
$$

and the parameter $\alpha$ is random variable or random vector, its probability distribution function is

$$
\alpha \sim G(\alpha) .
$$

Then the corresponding risk model is a mixed claim frequency risk model.

\section{Binomial-Poisson Model}

\subsection{The Setting-Up of the Model}

Definition 1. Suppose $M=\{M(t) ; t \geq 0\}$ is an homogeneous Poisson process with intensity $\lambda$. Consider $M(t)=m$; then the conditional distribution of $N=\{N(t) ; t \geq 0\}$ is a binomial 
distribution with parameters $m, p$. A sequence $\left\{Z_{k}\right\}_{1}^{\infty}$ is an independent and identically distributed nonnegative random variable, having the common distribution function $F$, with $F(0)=0$, mean value $\mu$, and variance $\sigma^{2}$; the above random process and random sequence are mutually independent; $c, p(c>0,0<p<1)$ are both constants.

Let

$$
X(t)=c t-\sum_{k=1}^{N(t)} Z_{k} .
$$

Then the process defined by (4) is a binomial-Poisson mixed claim frequency risk model.

The conditional moment generating function of $N$ is

$$
M_{N}(s \mid m)=\left(p e^{s}+q\right)^{m}, \quad(q=1-p) .
$$

Therefore

$$
\begin{aligned}
M_{N(t)}(s) & =E e^{s N(t)}=E\left[E\left(e^{s N(t)} \mid M(t)\right)\right] \\
& =\sum_{m=0}^{\infty}\left(p e^{s}+q\right)^{m} \frac{e^{-\lambda t}(\lambda t)^{m}}{m !} \\
& =\exp \left[\lambda p t\left(e^{s}-1\right)\right] .
\end{aligned}
$$

Then $\{N(t) ; t \geq 0\}$ is a homogeneous Poisson process with intensity $\lambda p$. Thus the model defined by (4) is a classical risk model.

2.2. The Meaning of the Model in the Insurance Practice. Suppose that $M(t)$ is the number of accidents during the interval $(0, t]$; the number of claims per accident $L$ is $0-1$ distribution with $p$; that is, $P(L=1)=p, P(L=0)=1-p$ (for example, in the deductible insurance, the probability of loss exceeding the amount of deductible is $p$ ); then the number of claims $N(t)$ during the interval $(0, t]$ is a conditional binomial distribution with parameters $m, p$. Obviously the process $\{N(t) ; t \geq 0\}$ is a $p$-sparse process of the process $\{M(t) ; t \geq 0\}$. $\{N(t) ; t \geq 0\}$ is a homogeneous Poisson process with intensity $\lambda p$, if only $\{M(t) ; t \geq 0\}$ is a homogeneous Poisson process with intensity $\lambda$. $c$ is the premium rate, $\left\{Z_{k}\right\}_{1}^{\infty}$ is the size of claim amount per accident, and $\sum_{k=1}^{N(t)} Z_{k}$ is the total claim process.

2.3. Several Conclusions about the Ultimate Ruin Probability. We can get easily

$$
E[X(t)]=(c-\lambda p \mu) t .
$$

In order to stabilize the operation of company, we should ensure that premiums received in a unit of time meet $c>\lambda p \mu$.

The relative safety loading $\rho$ is defined by

$$
\rho=\frac{c}{\lambda p \mu}-1
$$

We can now define the ruin probability $\psi(u)$ of a company facing the risk process (4) and having initial capital $u$. Consider

$$
\psi(u)=P\{u+X(t)<0 \text { for some } t>0\} .
$$

Let

$$
h(r)=\int_{0}^{\infty} e^{r z} d F(z)-1
$$

We assume that there exists $r_{\infty}>0$ such that $h(r) \uparrow+\infty$ when $r \uparrow r_{\infty}$, that is, a light-tailed distribution $F$.

Theorem 2. If $\rho>0, \psi(0)=\lambda p \mu / c$.

Theorem 3. Let amount claimed sequence $\left\{Z_{k}, k=1,2, \ldots\right\}$ with exponential distribution with mean $\mu$; then

$$
\psi(u)=\frac{1}{1+\rho} e^{-\rho u / \mu(1+\rho)},
$$

where $\rho$ is given by (8).

Theorem 4. Consider

$$
\lim _{u \longrightarrow \infty} e^{R u} \psi(u)=\frac{\rho \mu}{h^{\prime}(R)-c / \lambda p},
$$

where $R$ is the positive solution of $h(r)=c r / \lambda p$, which is called adjustment coefficient. And $\rho$ is given by (8).

Theorem 5. Ruin probability:

$$
\psi(u) \leq e^{-R u},
$$

where $R$ is the positive solution of $h(r)=c r / \lambda p$, which is called adjustment coefficient.

The above theorem can be derived directly by the corresponding results in classical risk theory [7].

\section{Poisson-Dualistic Model}

\subsection{The Setting-Up of the Model}

Definition 6. The random variable $\lambda$ follows two-point distribution; that is, $P\left(\lambda=\lambda_{i}\right)=p_{i}, i=1,2, p_{2}=1-p_{1}$. The conditional distribution of $N=\{N(t) ; t \geq 0\}$ about $\lambda$ is a homogeneous Poisson process with intensity $\lambda$. A sequence $\left\{Z_{k}\right\}_{1}^{\infty}$ is independent and identically distributed nonnegative random variable, having the common distribution function $F$, with $F(0)=0$, mean value $\mu$, and variance $\sigma^{2} ;\{N(t) ; t \geq 0\}$ and $\left\{Z_{k}\right\}$ are mutually independent; $p_{1}, p_{2}, \lambda_{1}, \lambda_{2}, c$ are both positive real constants.

Let

$$
Y(t)=c t-\sum_{k=1}^{N(t)} Z_{k} .
$$

Then the random process defined by (14) is a Poisson-dualistic mixed claim frequency risk model.

Obviously, $N=\{N(t) ; t \geq 0\}$ is a mixed Poisson process.

3.2. The Meaning of the Model in the Insurance Practice. We assume that a portfolio is composed of high risk and low risk insurance policy, where high risk policy is accounting for $p_{1}$ 
and low risk policy is accounting for $p_{2}=1-p_{1}$; the Poisson parameter of these two kinds of policy, respectively, is $\lambda_{1}, \lambda_{2}$ (corresponding high risk cover for $\lambda_{1}$, obviously $\lambda_{1}>\lambda_{2}$ ). $c$ is the premium rate, $\left\{Z_{k}\right\}_{1}^{\infty}$ is the size of claim amount per accident, and $\sum_{k=1}^{N(t)} Z_{k}$ is the total claim process.

3.3. Some Probabilistic Properties of Model. The probabilistic properties of the number of claims $N(t)$ are as follows.

Property 1. Claim frequency distribution is

$$
\begin{array}{r}
P(N(t)=k)=p_{1} \frac{e^{-\lambda_{1} t}\left(\lambda_{1} t\right)^{k}}{k !}+p_{2} \frac{e^{-\lambda_{2} t}\left(\lambda_{2} t\right)^{k}}{k !}, \\
k=0,1,2, \ldots .
\end{array}
$$

Proof. The above distribution can be derived by

$$
P(N(t)=k)=\int_{\Omega} P(N(t)=k \mid \lambda) d G(\lambda) .
$$

The proof is ended.

Property 2. The mean and variance of $N(t)$ are

$$
\begin{gathered}
E[N(t)]=\left(\lambda_{1} p_{1}+\lambda_{2} p_{2}\right) t \\
\operatorname{Var}[N(t)]=\left(\lambda_{1}-\lambda_{2}\right)^{2} p_{1} p_{2} t^{2}+\left(\lambda_{1} p_{1}+\lambda_{2} p_{2}\right) t .
\end{gathered}
$$

Proof. Consider

$$
\begin{aligned}
E[N(t)]= & E[E(N(t) \mid \lambda)]=E(\lambda t) \\
= & \left(\lambda_{1} p_{1}+\lambda_{2} p_{2}\right) t \\
\operatorname{Var}[N(t)]= & \operatorname{Var}[E(N(t) \mid \lambda)]+E[\operatorname{Var}(N(t) \mid \lambda)] \\
= & \operatorname{Var}(\lambda t)+E(\lambda t) \\
= & {\left[\lambda_{1}^{2} p_{1}+\lambda_{2}^{2} p_{2}-\left(\lambda_{1} p_{1}+\lambda_{2} p_{2}\right)^{2}\right] t^{2} } \\
& +\left(\lambda_{1} p_{1}+\lambda_{2} p_{2}\right) t \\
= & \left(\lambda_{1}-\lambda_{2}\right)^{2} p_{1} p_{2} t^{2}+\left(\lambda_{1} p_{1}+\lambda_{2} p_{2}\right) t
\end{aligned}
$$

The proof is ended.

Since the mean and variance of the Poisson distribution are always equal, if the sample variance of the portfolio's claim of a random variable is greater than the number of the sample mean, we can conclude that the existence of this policy combination of a degree of nonhomogeneity, and because $\operatorname{Var}[N(t)]-E[N(t)]=\operatorname{Var}(\lambda t)$ can reflect the variance of the degree of nonhomogeneity of portfolio, if the variance is much more greater than the mean, that is, the bigger $\left(\lambda_{1}-\lambda_{2}\right)^{2} p_{1} p_{2}$ is, the more serious the nonhomogeneity is.

Property 3. The moment generating function of $N(t)$ is

$$
M_{N(t)}(r)=p_{1} \exp \left[\lambda_{1} t\left(e^{r}-1\right)\right]+p_{2} \exp \left[\lambda_{2} t\left(e^{r}-1\right)\right] .
$$

Proof. Consider

$$
\begin{aligned}
M_{N(t)}(r) & =E e^{r N(t)} \\
& =E\left[E\left(e^{r N(t)} \mid \lambda\right)\right]=E\left[\exp \left(\lambda t\left(e^{r}-1\right)\right)\right] \\
& =p_{1} \exp \left[\lambda_{1} t\left(e^{r}-1\right)\right]+p_{2} \exp \left[\lambda_{2} t\left(e^{r}-1\right)\right] .
\end{aligned}
$$

The proof is ended.

Theorem 7. Assume that the random variable $\lambda$ follows twopoint distribution; that is, $P\left(\lambda=\lambda_{i}\right)=p_{i}, i=1,2$, and $p_{2}=$ $1-p_{1}$. The conditional distribution of $N=\{N(t) ; t \geq 0\}$ about $\lambda$ is a homogeneous Poisson process with intensity $\lambda$. Then the process $N$ has stationary increments.

Proof. Consider

$$
\begin{aligned}
P( & N(t+h)-N(t)=k) \\
& =E[P(N(t+h)-N(t)=k \mid \lambda)] \\
& =E\left[\frac{e^{-\lambda h}(\lambda h)^{k}}{k !}\right] \\
& =p_{1} \frac{e^{-\lambda_{1} h}\left(\lambda_{1} h\right)^{k}}{k !}+p_{2} \frac{e^{-\lambda_{2} h}\left(\lambda_{2} h\right)^{k}}{k !}, \quad k=0,1,2, \ldots
\end{aligned}
$$

The proof is ended.

Theorem 8. Assume that the random variable $\lambda$ follows twopoint distribution; that is, $P\left(\lambda=\lambda_{i}\right)=p_{i}, i=1,2, p_{2}=$ $1-p_{1}$. The conditional distribution of $N=\{N(t) ; t \geq 0\}$ about $\lambda$ is a homogeneous Poisson process with intensity $\lambda$ if and only if the interval $\left\{T_{n}\right\}$ of $\{N(t) ; t \geq 0\}$ about $\lambda$ has conditional independence and is the same as the exponential distribution with parameter $\lambda$; thus $\left\{T_{n}\right\}$ follow the following mixed exponential distribution:

$$
f_{T_{n}}(t)=p_{1} \lambda_{1} e^{-\lambda_{1} t}+p_{2} \lambda_{2} e^{-\lambda_{2} t}, \quad t>0 .
$$

Proof. We only need to prove that when the conditional distribution of $\left\{T_{n}\right\}$ about $\lambda$ is exponentially distributed, $\left\{T_{n}\right\}$ follow the mixed exponential distribution. Then, when $t>0$, the distribution function of $\left\{T_{n}\right\}$ is

$$
\begin{aligned}
F_{T_{n}}(t) & =P\left(T_{n} \leq t\right) \\
& =E\left[P\left(T_{n} \leq t \mid \lambda\right)\right]=E\left[1-e^{-\lambda t}\right] \\
& =p_{1}\left(1-e^{-\lambda_{1} t}\right)+p_{2}\left(1-e^{-\lambda_{2} t}\right) .
\end{aligned}
$$

Thus the density function of $\left\{T_{n}\right\}$ is

$$
f_{T_{n}}(t)=p_{1} \lambda_{1} e^{-\lambda_{1} t}+p_{2} \lambda_{2} e^{-\lambda_{2} t}, \quad t>0 .
$$

The proof is ended. 
Theorem 9. It occur $N(t)$ claims over $(0, t]$; then the occurrence probability of intensity $\lambda=\lambda_{1}$ for claim is

$$
P\left(\lambda=\lambda_{1} \mid N(t)=n\right)=\frac{p_{1} e^{-\lambda_{1} t}\left(\lambda_{1} t\right)^{n}}{p_{1} e^{-\lambda_{1} t}\left(\lambda_{1} t\right)^{n}+p_{2} e^{-\lambda_{2} t}\left(\lambda_{2} t\right)^{n}} .
$$

Proof. It can be derived by

$$
P\left(\lambda=\lambda_{1} \mid N(t)=n\right)=\frac{P\left(N(t)=n \mid \lambda=\lambda_{1}\right) P\left(\lambda=\lambda_{1}\right)}{P(N(t)=n)} .
$$

The proof is ended.

Thus we can get

$$
\begin{aligned}
P\left(\lambda=\lambda_{2} \mid N(t)=n\right) & =1-\frac{p_{1} e^{-\lambda_{1} t}\left(\lambda_{1} t\right)^{n}}{p_{1} e^{-\lambda_{1} t}\left(\lambda_{1} t\right)^{n}+p_{2} e^{-\lambda_{2} t}\left(\lambda_{2} t\right)^{n}} \\
& =\frac{p_{2} e^{-\lambda_{2} t}\left(\lambda_{2} t\right)^{n}}{p_{1} e^{-\lambda_{1} t}\left(\lambda_{1} t\right)^{n}+p_{2} e^{-\lambda_{2} t}\left(\lambda_{2} t\right)^{n}} .
\end{aligned}
$$

Theorem 10. Assume that $U(t)$ is the time interval from the moment $t$ to the next claim; then the conditional distribution of $U(t)$ is

$$
\begin{aligned}
& P(U(t) \leq x \mid N(t)=n) \\
& \quad=\frac{p_{1} e^{-\lambda_{1} t}\left(\lambda_{1} t\right)^{n}\left(1-e^{-\lambda_{1} x}\right)+p_{2} e^{-\lambda_{2} t}\left(\lambda_{2} t\right)^{n}\left(1-e^{-\lambda_{2} x}\right)}{p_{1} e^{-\lambda_{1} t}\left(\lambda_{1} t\right)^{n}+p_{2} e^{-\lambda_{2} t}\left(\lambda_{2} t\right)^{n}} .
\end{aligned}
$$

Proof. Obviously $U(t)$ is the occurrence time after the moment $t$, when $\lambda$ is a constant; then $U(t)$ follows the exponential distribution with $\lambda$. Then the result can be derived by

$$
\begin{aligned}
P( & (t) \leq x \mid N(t)=n) \\
= & P\left(U(t) \leq x \mid N(t)=n, \lambda=\lambda_{1}\right) \\
& \times P\left(\lambda=\lambda_{1} \mid N(t)=n\right) \\
& +P\left(U(t) \leq x \mid N(t)=n, \lambda=\lambda_{2}\right) \\
& \times P\left(\lambda=\lambda_{2} \mid N(t)=n\right) \\
= & \frac{p_{1} e^{-\lambda_{1} t}\left(\lambda_{1} t\right)^{n}\left(1-e^{-\lambda_{1} x}\right)+p_{2} e^{-\lambda_{2} t}\left(\lambda_{2} t\right)^{n}\left(1-e^{-\lambda_{2} x}\right)}{p_{1} e^{-\lambda_{1} t}\left(\lambda_{1} t\right)^{n}+p_{2} e^{-\lambda_{2} t}\left(\lambda_{2} t\right)^{n}} .
\end{aligned}
$$

The proof is ended.

The probabilistic properties of total amount of claims $S(t)=\sum_{k=1}^{N(t)} Z_{k}$ are as follows.
Property 4. The mean and variance of total amount of claims $S(t)=\sum_{k=1}^{N(t)} Z_{k}$ are

$$
\begin{gathered}
E[S(t)]=\left(\lambda_{1} p_{1}+\lambda_{2} p_{2}\right) \mu t \\
\operatorname{Var}[S(t)]=\mu^{2}\left(\lambda_{1}-\lambda_{2}\right)^{2} p_{1} p_{2} t^{2} \\
+\left(\mu^{2}+\sigma^{2}\right)\left(\lambda_{1} p_{1}+\lambda_{2} p_{2}\right) t .
\end{gathered}
$$

Proof. Consider

$$
\begin{aligned}
E[S(t)]= & E[N(t)] E Z_{k}=\left(\lambda_{1} p_{1}+\lambda_{2} p_{2}\right) \mu t \\
\operatorname{Var}[S(t)]= & \operatorname{Var}[E(S(t) \mid N(t))]+E[\operatorname{Var}(S(t) \mid N(t))] \\
= & \operatorname{Var}\left[N(t) E Z_{k}\right]+E\left[N(t) \operatorname{Var} Z_{k}\right] \\
= & \mu^{2} \operatorname{Var}[N(t)]+\sigma^{2} E[N(t)] \\
= & \mu^{2}\left[\left(\lambda_{1}-\lambda_{2}\right)^{2} p_{1} p_{2} t^{2}+\left(\lambda_{1} p_{1}+\lambda_{2} p_{2}\right) t\right] \\
& +\sigma^{2}\left(\lambda_{1} p_{1}+\lambda_{2} p_{2}\right) t \\
= & \mu^{2}\left(\lambda_{1}-\lambda_{2}\right)^{2} p_{1} p_{2} t^{2} \\
& +\left(\mu^{2}+\sigma^{2}\right)\left(\lambda_{1} p_{1}+\lambda_{2} p_{2}\right) t .
\end{aligned}
$$

The proof is ended.

Property 5. The moment generating function of total amount of claims $S(t)=\sum_{k=1}^{N(t)} Z_{k}$ is

$$
\begin{aligned}
M_{S(t)}(r)= & p_{1} \exp \left[\lambda_{1} t\left(M_{Z}(r)-1\right)\right] \\
& +p_{2} \exp \left[\lambda_{2} t\left(M_{Z}(r)-1\right)\right],
\end{aligned}
$$

where $M_{Z}(r)$ is the moment generation function of the individual claim amount.

Proof. We can get the following from (19):

$$
\begin{aligned}
M_{S(t)}(r)= & E e^{r S(t)}=E\left[\left(M_{Z}(r)\right)^{N(t)}\right] \\
= & M_{N(t)}\left(\ln M_{Z}(r)\right) \\
= & p_{1} \exp \left[\lambda_{1} t\left(M_{Z}(r)-1\right)\right] \\
& +p_{2} \exp \left[\lambda_{2} t\left(M_{Z}(r)-1\right)\right] .
\end{aligned}
$$

The proof is ended.

3.4. Estimation of Lundberg Exponential Upper Bounds for the Ultimate Ruin Probability. Obviously, $E[Y(t)]=\left[c-\left(\lambda_{1} p_{1}+\right.\right.$ $\left.\left.\lambda_{2} p_{2}\right) \mu\right] t$.

It seems very natural to assume the premium rate per unit of time $c>\left(\lambda_{1} p_{1}+\lambda_{2} p_{2}\right) \mu$. Further, let $c>\lambda_{1} \mu$, in order to stabilize the operation of the company.

The relative safety loading:

$$
\rho=\frac{c}{\left(\lambda_{1} p_{1}+\lambda_{2} p_{2}\right) \mu}-1 .
$$


Obviously,

$$
\rho>\frac{c}{\lambda_{1} \mu}-1 \hat{=} \rho_{1}
$$

$\rho_{1}$ can be seen as the corresponding relative safety loading of portfolio consisted by high risk policy. Obviously in terms of premium rate and the average individual claim amount are equal, the relative safety loading of the corresponding portfolio of model (14) should be greater.

Let $u$ be the initial capital; then the ruin moment is defined as

$$
T_{u}=\inf \{t \geq 0 \mid u+Y(t)<0\} .
$$

Obviously $T_{u}$ is a $F^{Y}$-stopping time.

The finite time ruin probability is

$$
\psi(u, t)=P\left(T_{u}<t\right) .
$$

The ultimate ruin probability is

$$
\psi(u)=P\left(T_{u}<\infty\right) .
$$

Let

$$
h(r)=M_{Z}(r)-1 .
$$

We assume that there exists $r_{\infty}>0$, such that $h(r) \uparrow+\infty$ when $r \uparrow r_{\infty}$. It is easy to be seen that $h(0)=0, h^{\prime}(r)>0$, and $h^{\prime \prime}(r)>0$ and that $h(r)$ is continuous on $\left[0, r_{\infty}\right.$ ) (where $\left.r_{\infty} \leq+\infty\right)$.

Easy to verify, the risk process defined by (14) is a right continuous process and has the following properties:

(i) $Y(0)=0 \quad P-$ a.s.;

(ii) $Y$ has stationary and independent increments about $\lambda$;

(iii) $E[Y(t)]=\left[c-\left(\lambda_{1} p_{1}+\lambda_{2} p_{2}\right) \mu\right] t>0$.

Let

$$
F_{\infty}^{\lambda}=\sigma(\lambda, t \geq 0), \quad F_{t}=F_{\infty}^{\lambda} \vee F_{t}^{Y} .
$$

Note that $F_{0}=F_{\infty}^{\lambda}$.

We can easily get

$$
\begin{aligned}
E\left[e^{-r Y(t)} \mid F_{\infty}^{\lambda}\right] & =E\left\{\exp [-r(c t-S(t))] \mid F_{\infty}^{\lambda}\right\} \\
& =\exp (-r c t) \cdot E\left[\exp (r S(t)) \mid F_{\infty}^{\lambda}\right] \\
& =\exp [t(\lambda h(r)-r c)] .
\end{aligned}
$$

From the hypothesis of function $h(r)$, we know $\exists r>0$, s.t. $E\left[e^{-r Y(t)} \mid F_{\infty}^{\lambda}\right]<\infty$.

From property (ii) of $Y$, we have

$$
\begin{aligned}
\exp [t(\lambda h(r)-r c)] & =E\left[e^{-r Y(t)} \mid F_{\infty}^{\lambda}\right] \\
& =E\left[e^{-r(Y(t)-Y(s))} \cdot e^{-r Y(s)} \mid F_{\infty}^{\lambda}\right] \\
& =E\left[e^{-r(Y(t)-Y(s))} \mid F_{\infty}^{\lambda}\right] E\left[e^{-r Y(s)} \mid F_{\infty}^{\lambda}\right] .
\end{aligned}
$$

Thus

$$
E\left[e^{-r(Y(t)-Y(s))} \mid F_{\infty}^{\lambda}\right]=\exp [(\lambda h(r)-r c)(t-s)] .
$$

Theorem 11. Let

$$
M_{u}(t)=\frac{\exp [-r(u+Y(t))]}{\exp [t(\lambda h(r)-r c)]} .
$$

Thus $M_{u}=\left\{M_{u}(t) ; t \geq 0\right\}$ is an F-martingale.

Proof. We get from (43) that

$$
\begin{aligned}
& E\left[M_{u}(t) \mid F_{s}\right] \\
& =E\left[\frac{\exp (-r(u+Y(t)))}{\exp (t(\lambda h(r)-r c))} \mid F_{s}\right] \\
& =E\left[\frac{\exp (-r(u+Y(s)))}{\exp (s(\lambda h(r)-r c))} \cdot \frac{\exp (-r(Y(t)-Y(s)))}{\exp [(\lambda h(r)-r c)(t-s)]} \mid F_{s}\right] \\
& =M_{u}(s) E\left[\frac{\exp (-r(Y(t)-Y(s)))}{\exp [(\lambda h(r)-r c)(t-s)]} \mid F_{s}\right] \\
& =M_{u}(s) .
\end{aligned}
$$

The proof is ended.

Choose $t_{0}<\infty$ and consider $t_{0} \wedge T_{u}$ which is a bounded $F$-stopping time. We get from Doob's stopping theorem that

$$
\begin{aligned}
e^{-r u}= & M_{u}(0)=E^{F_{0}}\left[M_{u}\left(t_{0} \wedge T_{u}\right)\right] \\
= & E^{F_{0}}\left[M_{u}\left(t_{0} \wedge T_{u}\right) \mid T_{u} \leq t_{0}\right] P^{F_{0}}\left(T_{u} \leq t_{0}\right) \\
& +E^{F_{0}}\left[M_{u}\left(t_{0} \wedge T_{u}\right) \mid T_{u}>t_{0}\right] P^{F_{0}}\left(T_{u}>t_{0}\right) \\
\geq & E^{F_{0}}\left[M_{u}\left(t_{0} \wedge T_{u}\right) \mid T_{u} \leq t_{0}\right] P^{F_{0}}\left(T_{u} \leq t_{0}\right) \\
= & E^{F_{0}}\left[M_{u}\left(T_{u}\right) \mid T_{u} \leq t_{0}\right] P^{F_{0}}\left(T_{u} \leq t_{0}\right) .
\end{aligned}
$$

Using $u+Y\left(T_{u}\right) \leq 0$ on $\left\{T_{u}<\infty\right\}$, the lower bound was shown to be given by

$$
\begin{aligned}
E^{F_{0}} & {\left[M_{u}\left(T_{u}\right) \mid T_{u} \leq t_{0}\right] } \\
& =E^{F_{0}}\left[\frac{\exp \left(-r\left(u+Y\left(T_{u}\right)\right)\right)}{\exp \left(T_{u}(\lambda h(r)-r c)\right)} \mid T_{u} \leq t_{0}\right] \\
& \geq E^{F_{0}}\left[\frac{1}{\exp \left(T_{u}(\lambda h(r)-r c)\right)} \mid T_{u} \leq t_{0}\right] \\
& \geq \inf _{0 \leq t \leq t_{0}}\left[\frac{1}{\exp (t(\lambda h(r)-r c))}\right] .
\end{aligned}
$$

Thus we have

$$
\begin{aligned}
P^{F_{0}}\left(T_{u} \leq t_{0}\right) & \leq \frac{e^{-r u}}{E^{F_{0}}\left[M_{u}\left(T_{u}\right) \mid T_{u} \leq t_{0}\right]} \\
& \leq e^{-r u} \sup _{0 \leq t \leq t_{0}}\{\exp [t(\lambda h(r)-r c)]\} .
\end{aligned}
$$


and, by taking expectation,

$$
\begin{aligned}
\psi\left(u, t_{0}\right) & =P\left(T_{u} \leq t_{0}\right) \\
& \leq e^{-r u} E\left\{\sup _{0 \leq t \leq t_{0}} \exp [t(\lambda h(r)-r c)]\right\} .
\end{aligned}
$$

When $t_{0} \longrightarrow \infty$ in the above equation, we get

$$
\psi(u) \leq e^{-r u} E\left\{\sup _{t \geq 0} \exp [t(\lambda h(r)-r c)]\right\} .
$$

We now want to choose $r$ as large as possible under the restriction $E\left\{\sup _{t \geq 0} \exp [t(\lambda h(r)-r c)]\right\}<\infty$. Let $R$ denote that value, named adjustment coefficient of surplus process (14).

Since

$$
\begin{gathered}
E\left\{\sup _{t \geq 0} \exp [t(\lambda h(r)-r c)]\right\} \\
=p_{1} \sup _{t \geq 0} \exp \left[t\left(\lambda_{1} h(r)-r c\right)\right] \\
+p_{2} \sup _{t \geq 0} \exp \left[t\left(\lambda_{2} h(r)-r c\right)\right] \\
=p_{1} \sup _{t \geq 0} e^{t g_{1}(r)}+p_{2} \sup _{t \geq 0} e^{t g_{2}(r)},
\end{gathered}
$$

where $g_{i}(r)=\lambda_{i} h(r)-r c, i=1,2$, the two terms of the right side in the above equality are both positive, which corresponds to restrict

$$
g_{1}(r) \leq 0, \quad g_{2}(r) \leq 0 .
$$

Since

$$
g_{1}(r) \geq g_{2}(r),
$$

thus we just need to restrict

$$
g_{1}(r) \leq 0 .
$$

Then we have

$$
R=\sup \left\{r \mid g_{1}(r) \leq 0\right\} .
$$

Theorem 12. The ultimate ruin probability meets the inequality

$$
\psi(u) \leq e^{-R u},
$$

where $R$ is the only positive solution of $g_{1}(r)=0$, named adjustment coefficient.

Proof. For $g_{1}(r)=\lambda_{1} h(r)-r c$, we have

$$
g_{1}(0)=0, \quad g_{1}^{\prime}(r)=\lambda_{1} h^{\prime}(r)-c .
$$

Therefore

$$
g_{1}^{\prime}(0)=\lambda_{1} h^{\prime}(0)-c=\lambda_{1} \mu-c<0 .
$$

Then there exists small enough $\Delta r \in\left(0, r_{\infty}\right)$ such that $g_{1}(\Delta r)<0$. Further $g_{1}(r) \longrightarrow+\infty$ when $r_{\infty}<+\infty$ and $r \uparrow r_{\infty}$.

When $r_{\infty}=+\infty$, since $M_{Z}(r)=1+r \mu_{1}+r^{2} \mu_{2} / 2+\cdots>$ $r^{2} \mu_{2} / 2$, where $\mu_{j}$ is the $j$ th moment of the claim amount $\left\{Z_{k}\right\}$, then

$$
\begin{aligned}
g_{1}(r) & =\lambda_{1} M_{Z}(r)-\lambda_{1}-r c \\
& >\frac{\lambda_{1} \mu_{2}}{2} r^{2}-\lambda_{1}-r c \longrightarrow+\infty, \quad(r \longrightarrow+\infty) .
\end{aligned}
$$

Thus there must exist $r^{*} \in\left(0, r_{\infty}\right)$ such that $g_{1}\left(r^{*}\right)=0$. And from $g_{1}^{\prime \prime}(r)=\lambda_{1} h^{\prime \prime}(r)>0$, we know $g_{1}(r)$ is a lower convex function.

Then $g_{1}(r)<0$ for $0<r<r^{*}$ and $g_{1}(r)>0$ for $r^{*}<r<$ $r_{\infty}$.

Therefore there exists the only positive solution of $g_{1}(r)=$ 0 ; let $r^{*}$ denote that value. From (55) we know $R=r^{*}$, so we get $\psi(u) \leq e^{-R u}$.

The proof is ended.

3.5. Boundary of the Adjustment Coefficient R. The exact value of adjustment coefficient $R$ generally cannot be determined by $g_{1}(r)=0$, but because of its great significance to estimate the upper bound of the ruin probability, we estimate the boundary of the adjustment coefficient $R$.

Theorem 13. The adjustment coefficient $R$ meets the inequality

$$
R<\frac{2\left(c-\lambda_{1} \mu\right)}{\lambda_{1}\left(\mu^{2}+\sigma^{2}\right)} .
$$

Proof. From [7] we know $R<2\left(c-\lambda_{1} \mu\right) / \lambda_{1} \mu_{2}$; then we can get the conclusion.

The proof is ended.

Form the above theorem and the expression of the relative safety loadings $\rho$, $\rho_{1}$, we get

$$
R<\frac{2\left(c-\lambda_{1} \mu\right)}{\lambda_{1}\left(\mu^{2}+\sigma^{2}\right)}=\frac{2 \rho_{1} \lambda_{1} \mu}{\lambda_{1}\left(\mu^{2}+\sigma^{2}\right)}=\frac{2 \rho_{1} \mu}{\mu^{2}+\sigma^{2}}<\frac{2 \rho \mu}{\mu^{2}+\sigma^{2}} .
$$

The result shows that the upper bound of $R$ can be only defined by the one- or two-order moment of individual claim amount and the relative safety loading; while having nothing to do with the Poisson parameter, this case have its convenience in use.

Theorem 14 (see [7]). If the individual claim amount $\left\{Z_{k}\right\}$ has the upper bound $W$, that is, $Z_{k} \leq W$, we have

$$
R>\frac{1}{W} \ln \frac{c}{\lambda_{1} \mu}=\frac{1}{W} \ln \left(1+\rho_{1}\right) .
$$

The result indicates that, under the conditions of the theorem, the lower bound of $R$ can be only defined by the relative safety loading $\rho_{1}$ and the upper bound of individual claim amount. 
When the exact value of $R$ cannot be determined by the equation $g_{1}(r)=0$, we can solve its approximate solution by the numerical method, and using these two boundaries as the initial values for iteration, we can quickly find the approximate solution satisfying requirements of certain accuracy.

3.6. The Probability of Survival. Except for a few special cases, generally, complicated calculation is needed to get the adjustment coefficient values. Through the following discussion on integrodifferential equations satisfied by survival probability, we can avoid the computation of the adjustment coefficient. bility.

Let $\Phi(u)=1-\psi(u)$; then $\Phi(u)$ indicates survival proba-

Since the number of claim process $\{N(t) ; t \geq 0\}$ about $\lambda$ is a conditional renewal process and will not be ruined during the period of $\left(0, S_{1}\right)$, then we get

$$
\begin{aligned}
& \Phi(u) \\
& =E\left[P\left(Y(t) \geq 0, \forall t \geq 0 \mid S_{1}, Z_{1}, \lambda\right)\right] \\
& =E\left\{E\left[\Phi\left(u+c S_{1}-Z_{1}\right) \mid \lambda\right]\right\} \\
& =E\left\{\int_{0}^{\infty} \lambda e^{-\lambda t} \int_{0}^{u+c t} \Phi(u+c t-z) d F(z) d t\right\} \\
& =\int_{0}^{\infty}\left(p_{1} \lambda_{1} e^{-\lambda_{1} t}+p_{2} \lambda_{2} e^{-\lambda_{2} t}\right) \int_{0}^{u+c t} \Phi(u+c t-z) d F(z) d t .
\end{aligned}
$$

Let

$$
x=u+c t .
$$

Then we have

$$
\begin{aligned}
& \Phi(u) \\
& =\frac{p_{1} \lambda_{1}}{c} e^{\lambda_{1} u / c} \int_{u}^{\infty} e^{-\lambda_{1} x / c} \int_{0}^{x} \Phi(x-z) d F(z) d x \\
& \quad+\frac{p_{2} \lambda_{2}}{c} e^{\lambda_{2} u / c} \int_{u}^{\infty} e^{-\lambda_{2} x / c} \int_{0}^{x} \Phi(x-z) d F(z) d x .
\end{aligned}
$$

Let two terms of the hand side in the above equality be, respectively, $\Phi_{1}(u)$ and $\Phi_{2}(u)$; then $\Phi(u)=\Phi_{1}(u)+\Phi_{2}(u)$.

Theorem 15. The integrodifferential equations assured by $\Phi_{1}(u)$ and $\Phi_{2}(u)$ are

$$
\begin{aligned}
\Phi_{1}^{\prime}(u)= & \frac{\lambda_{1}}{c} \Phi_{1}(u) \\
& -\frac{p_{1} \lambda_{1}}{c} \int_{0}^{u}\left[\Phi_{1}(u-z)+\Phi_{2}(u-z)\right] d F(z) \\
\Phi_{2}^{\prime}(u)= & \frac{\lambda_{2}}{c} \Phi_{2}(u) \\
& -\frac{p_{2} \lambda_{2}}{c} \int_{0}^{u}\left[\Phi_{1}(u-z)+\Phi_{2}(u-z)\right] d F(z) .
\end{aligned}
$$

Proof. From (65) and the hypothesis of $\Phi_{1}(u)$ and $\Phi_{2}(u)$, by taking derivation, we can get the above result.

The proof is ended.
$\Phi_{1}(u)$ and $\Phi_{2}(u)$ are calculated using the above equations; thus the expression of $\Phi(u)$ can be obtained. According to the above method to obtain the survival probability, we can immediately get the expression of ruin probability, and then the calculation of the adjustment coefficient can be avoided.

\section{Conflict of Interests}

The authors declare that there is no conflict of interests regarding the publication of this paper.

\section{Acknowledgments}

This work was jointly supported by the National Natural Science Foundation of China under Grant no. 11101053, the China Postdoctoral Science Foundation under Grant no. 20140550097, the Key Project of Chinese Ministry of Education under Grant no. 211118, the Hunan Provincial NSF under Grant no. 11JJ1001, and the Scientific Research Funds of Hunan Provincial Science and Technology Department of China under Grant no. 2013SK3143.

\section{References}

[1] A. Anandkumar, D. Hsu, and S. M. Kakade, "A method of moments for mixture models and hidden Markov models," http://arxiv.org/abs/1203.0683.

[2] H. Albrecher and V. Lautscham, "From ruin to bankruptcy for compound poisson surplus processes," ASTIN Bulletin, vol. 43, no. 2, pp. 213-243, 2013.

[3] H. U. Gerber, E. S. W. Shiu, and Y. Hailiang, "The Omega model: from bankruptcy to occupation times in the red," European Actuarial Journal, vol. 2, no. 2, pp. 259-272, 2012.

[4] V. Melnykov and R. Maitra, "Finite mixture models and modelbased clustering," Statistics Surveys, vol. 4, pp. 80-116, 2010.

[5] N. Städler, P. Bühlmann, and S. van de Geer, " $\ell$ 1-penalization for mixture regression models," Test, vol. 19, no. 2, pp. 209-256, 2010.

[6] F. Xiong, "Some results on the compound claim frequency model and mixedl claim frequency model," Mathematics in Economics, vol. 20, no. 1, pp. 48-54, 2003.

[7] G. Jan, Aspects of Risk Theory, Springer, New York, NY, USA, 1991. 


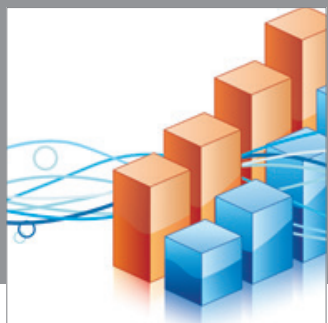

Advances in

Operations Research

mansans

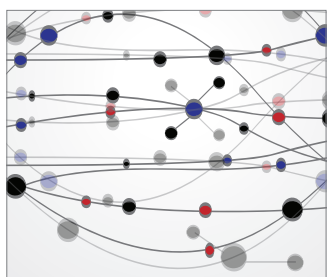

The Scientific World Journal
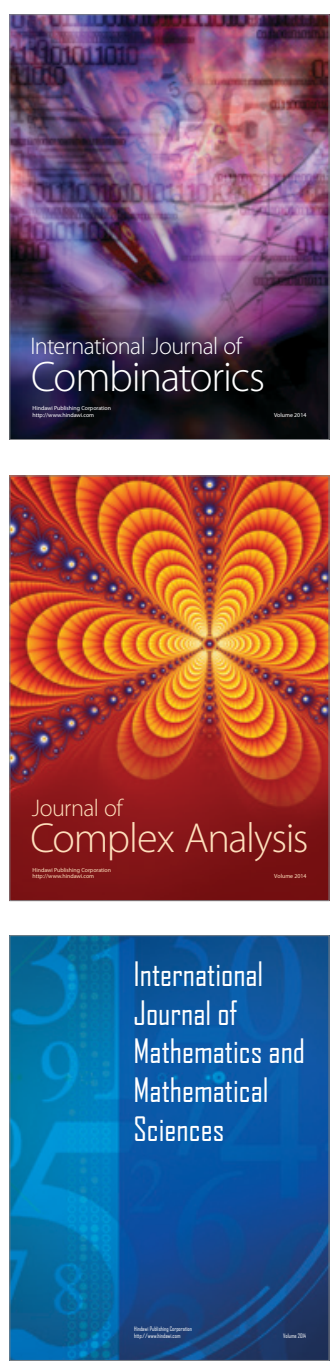
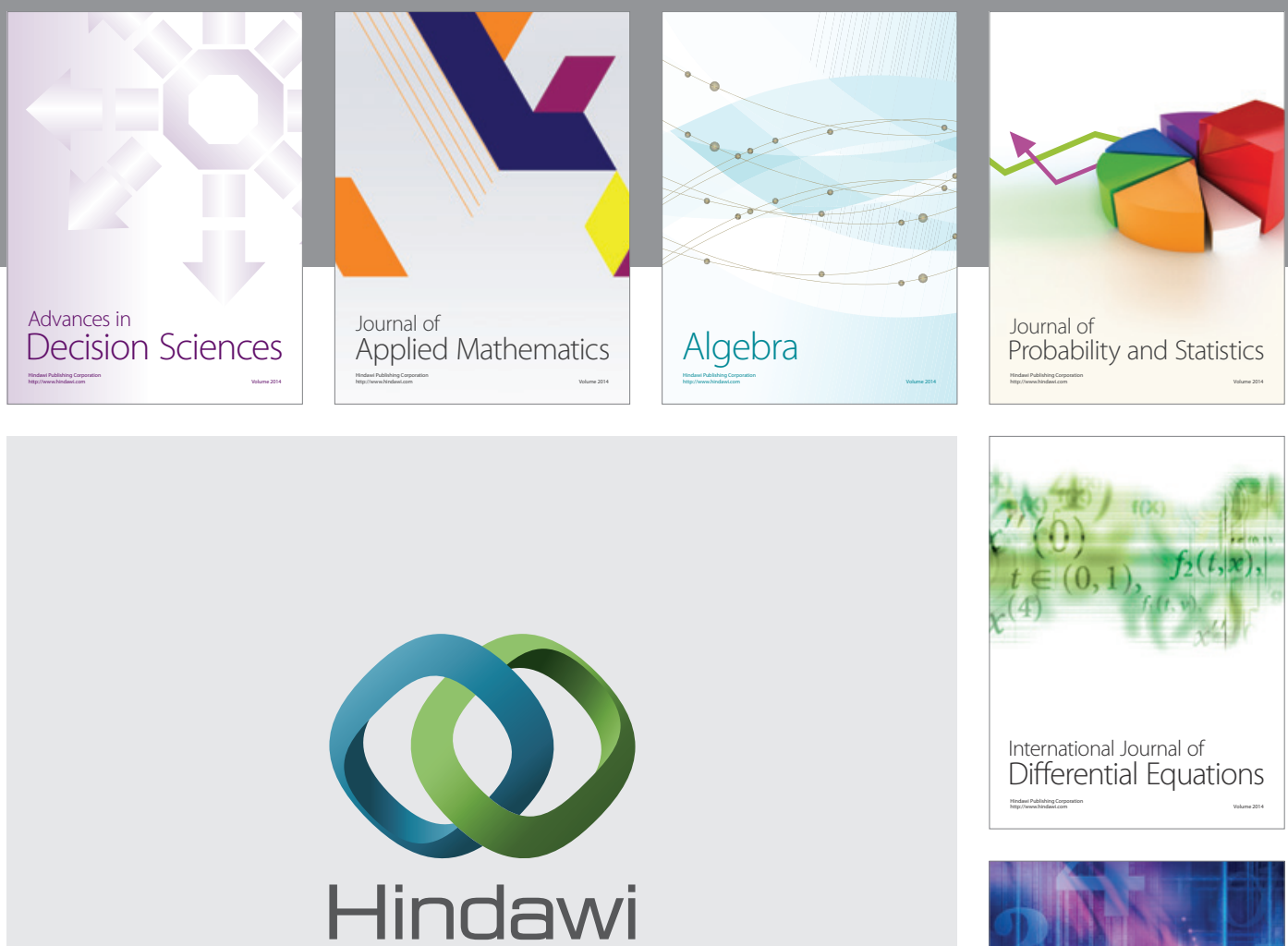

Submit your manuscripts at http://www.hindawi.com


Journal of

Function Spaces

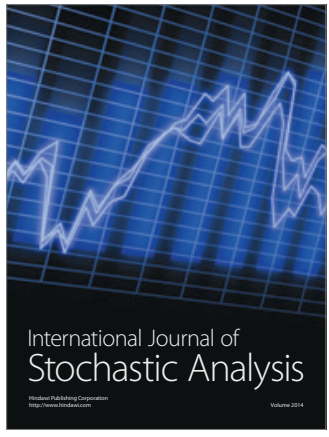

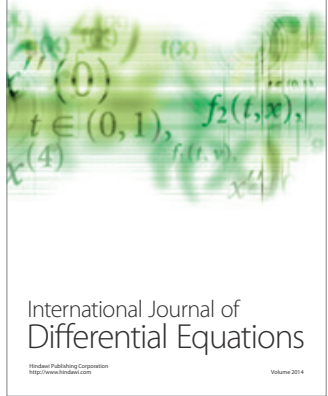
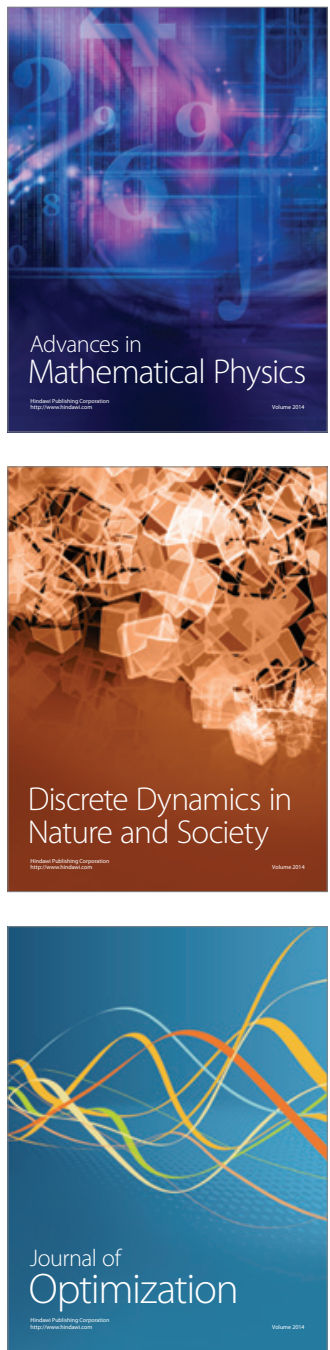